\title{
Hydrus Microstent
}

\author{
Panagiotis Laspas and Norbert Pfeiffer
}

\section{$5.1 \quad$ Introduction}

The Hydrus Microstent (Ivantis Inc., Irvine, CA, USA) is an intracanalicular scaffold which reduces the intraocular pressure (IOP) in order to treat glaucoma. The Microstent comes preloaded in a delivery system designed for ab-interno implantation under gonioscopic visualization. This is performed through the trabecular meshwork into Schlemm's canal typically in conjunction with planned cataract surgery. The implantation procedure is relatively intuitive and, if combined with cataract surgery, is usually performed at the end of surgery through the same corneal incision [1].

\subsection{Material/Design}

The Hydrus Microstent has a length of $8 \mathrm{~mm}$. A 7-mm scaffold segment resides within the lumen of Schlemm's canal and a 1-mm inlet portion resides within the anterior chamber (Fig. 5.1) [1]. The Microstent is designed to fit the curvature of the canal without obstructing collector channel ostia located along the posterior wall (Fig. 5.2) [2]. The 8-mm Microstent is a modification of an earlier design that was $15 \mathrm{~mm}$ in length with a larger, nearly circular profile [3].

The Hydrus Microstent is made of nitinol (nickel-titanium alloy), a material with unique shape memory properties, which has been used widely in vascular medicine and other medical applications $[4,5]$. The biocompatibility of nitinol for ocular applications has been reported previously and the Hydrus Microstent was initially evaluated in rabbit and primate ocular models $[6,7]$.

P. Laspas $(\bowtie) \cdot$ N. Pfeiffer

Department of Ophthalmology, University Medical Center, Johannes Gutenberg University

Mainz, Mainz, Germany

e-mail: Panagiotis.Laspas@unimedizin-mainz.de 
Fig. 5.1 After implantation into the Schlemm's canal, the Hydrus ${ }^{\circledR}$ Microstent is visible through slit lamp gonioscopic examination. About $7 \mathrm{~mm}$ of the Microstent lies in Schlemm's canal, scaffolding and dilating it. A smaller segment of the Microstent (about $1 \mathrm{~mm}$ ) prolapses at the site of implantation through the trabecular meshwork back in the anterior chamber. (Copyright permission granted by Elsevier Inc. according to STM Guidelines [1])
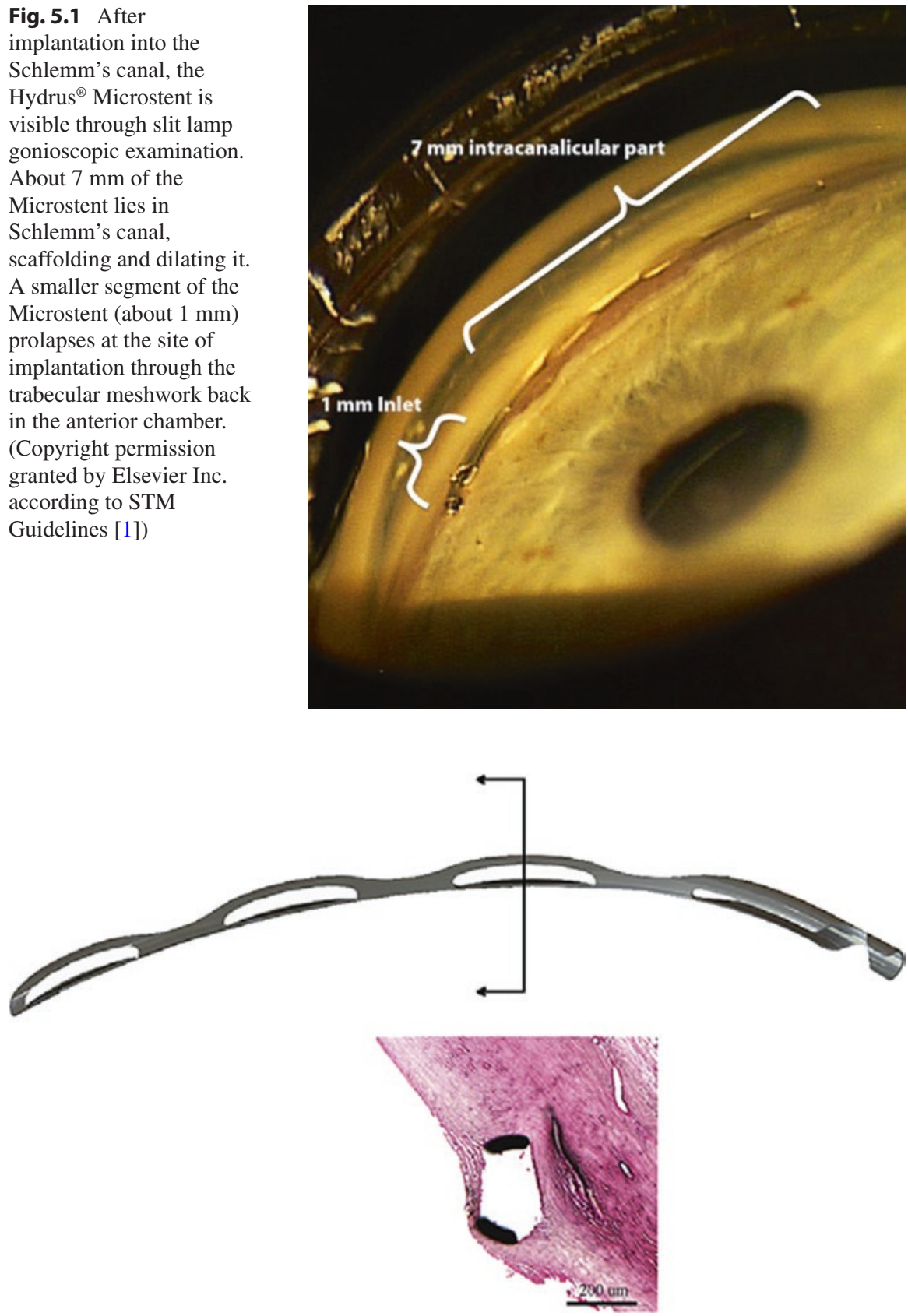

Fig. 5.2 The 8-mm Hydrus ${ }^{\circledR}$ Microstent consists of a scaffold of three windows and three spines and an inlet region. The cross section of the Hydrus Microstent in the Schlemm's canal of a donor eye shows the canal dilated, the trabecular meshwork stretched, and the access to collector channel ostia free. (Copyright permission granted by Elsevier Inc. according to STM Guidelines [2]) 


\subsubsection{Mechanism of Action}

The Hydrus Microstent has a dual mode of action: First, this is a trabecular bypass minimally invasive glaucoma surgery (MIGS) device: the trabecular resistance, which plays a major role in the pathogenesis of open-angle glaucoma, is bypassed as aqueous gains direct access to the Schlemm's canal through the small inlet portion. Secondly, it dilates and scaffolds Schlemm's canal in order to increase the circumferential flow and maintain or facilitate access to collector channels (Fig. 5.3). Surgical procedures, such as canaloplasty, are based on a similar principle [8]. It has been hypothesized following ex vivo studies that elevated IOP itself may lead to alterations in the anatomy of trabecular meshwork and Schlemm's canal, which then becomes narrower or collapses [9]. The surgical dilation of Schlemm's canal leads to increased aqueous outflow and a reduction in IOP [10]. While canaloplasty offers a $360^{\circ}$ dilation, the Hydrus Microstent can dilate only part of the canal's circumference. It creates a maximum Schlemm's canal dilation of $241 \mu \mathrm{m}$ or approximately four to five times the natural cross-sectional area of the canal across its length [3].

A mathematical model has demonstrated that bypassing the trabecular meshwork increases the pressure and circumferential flow rate within Schlemm's canal, as well as the flow rate in collector channels adjacent to the bypass [11]. The same model showed that dilating Schlemm's canal adjacent to the bypass further reduces the pressure in the dilated region, which increases the circumferential flow rate even more. Thus, the Hydrus Microstent is unique in its design, which allows aqueous to flow without significant resistance from the anterior chamber directly into the collector channels. The small proximal 1-mm inlet of the Microstent in the anterior chamber bypasses the trabecular meshwork and permits a direct pathway for aqueous flow from the anterior chamber to Schlemm's canal, while the larger

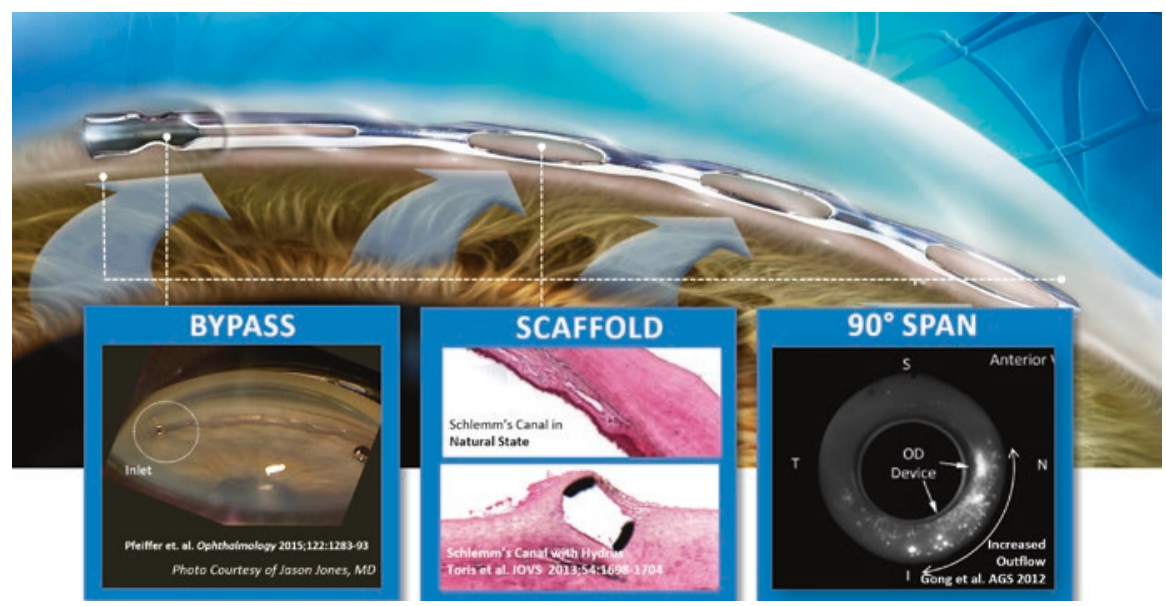

Fig. 5.3 The Hydrus ${ }^{\circledR}$ Microstent delivery system. (Copyright Ivantis Inc., Irvine, CA, USA; reproduced with permission) 
intracanalicular portion provides persistent dilation of Schlemm's canal and access to the collector channel ostia.

\subsection{Ex Vivo Testing}

Grierson et al. reported the histological changes in primate and rabbit eyes after implantation of the Hydrus Microstent [7]. The host response to the Microstent within the ocular tissues at the anterior chamber angle was judged to be minimal: there was no evidence of tissue degeneration near the Microstent or elsewhere within the eyes. No histopathological signs of metallosis such as depigmentation, apoptosis, or tissue necrosis were present and there was no evidence of significant intraocular inflammation. A low-grade mononuclear immune response involving a few scattered macrophages was present in some tissues both close to and remote from the Microstent. An extremely thin capsule wall was found around the Microstent, consisting of one to two thin spindle-shaped fibroblasts without a substantial fibrous collagen component.

Johnstone et al. used scanning electron microscopy to assess the structure of the outer wall of Schlemm's canal after Microstent implantation in human cadaveric eyes [2]. Particulate debris was found at the site of the Microstent but did not occlude Schlemm's canal. Collector channels were regularly visible with intact margins and were not obstructed or compressed.

The mechanism of action of the device was tested in anterior segment perfusion models using human donor eyes [3, 12, 13]. Gulati et al. and Hays et al. performed experiments using the $8 \mathrm{~mm}$ version of the Microstent, while Camras et al. tested the effect of the previous $15 \mathrm{~mm}$ version. Overall, these studies confirmed an increase in outflow facility, compared with controls after sham treatment. This increase in outflow facility was more profound when the IOP was elevated. This implies that eyes with higher outflow resistance can be expected to have a greater improvement in outflow and therefore a greater decrease in IOP with the Hydrus Microstent. In addition, it is possible that the Microstent prevented Schlemm's canal from closure and collapse when the IOP was elevated, a phenomenon which has been shown in studies of the anatomy of the angle tissues in eyes with raised IOP [9, 14]. The dilation of Schlemm's canal by the Hydrus Microstent enabled the aqueous outflow to be measurably higher in eyes with significant IOP elevation, even though only one-quarter of the entire Schlemm's canal was stented. Another interesting finding in the study of Camras et al. [12] was that the improved outflow was attributable only to the presence of the Microstent itself and not by the implantation process or the subsequent histological changes in the trabecular meshwork or the Schlemm's canal: the outflow was not improved in eyes in which the Microstent was first implanted and then removed again. Histological examination of the site of implantation offered a possible explanation. It showed that the Schlemm's canal was widened by the Microstent, while the overlying trabecular meshwork appeared stretched but intact. Thus, damage to the angle structures by Hydrus Microstent implantation or the Microstent itself was very mild and was not likely to reduce trabecular resistance. 
Fig. 5.4 The trimodal mechanism of action of the Hydrus ${ }^{\circledR}$ Microstent. (Copyright Ivantis Inc., Irvine, CA, USA; reproduced with permission)

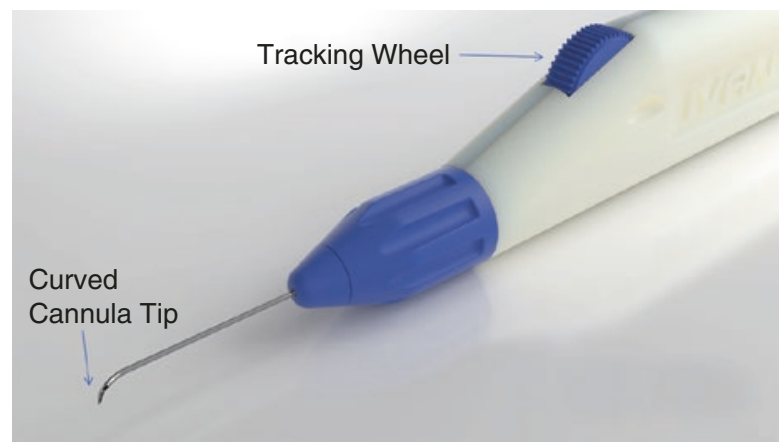

\subsection{Surgical Technique}

The Hydrus Microstent comes preloaded in a sterile delivery cannula (Fig. 5.4). The cannula is slightly curved so as to conform to the morphology of the angle and the Schlemm's canal. During implantation, the Hydrus Microstent is fed along 3 clock hours of Schlemm's canal. In order to facilitate this, the cannula can be rotated on the injector, permitting the surgeon to optimize his or her hand orientation for implantation. The device is inserted into the anterior chamber through a small clearcorneal paracentesis. Visualization of the angle via a goniolens is required for the precise implantation of the Hydrus Microstent. The cannula penetrates the trabecular meshwork at a very small angle almost tangential to its surface, so as to cannulate Schlemm's canal. Subsequently, the tracking wheel on the delivery system is used to slowly advance and implant the Hydrus Microstent, while keeping the cannula tip firmly in place. When approximately $1 \mathrm{~mm}$ of the proximal Microstent protrudes as an inlet from the trabecular meshwork into the anterior chamber, the delivery system is fully released.

\subsection{Site of Implantation}

The most common site for the implantation of the Hydrus Microstent is the nasal quadrant due to its accessibility through a clear corneal temporal incision [15]. The nasal angle also has the highest concentration of collector channels [16]. However, bypassing the trabecular meshwork may not be sufficient for lowering the IOP in some eyes, as the distal conventional aqueous outflow pathway, such as the collector channels and aqueous veins, may be obstructed and may also confer significant resistance to aqueous outflow. Correct identification of the parts of the angle with functional collector channels and targeted implantation of the Hydrus Microstent could theoretically improve the efficacy of the stent in lowering IOP.

The intraoperative signs which indicate the success of $a b$-interno trabeculotomy can also be used to assess whether the Hydrus Microstent has been implanted correctly [17]. For example, the presence of an episcleral venous fluid wave (blanching 
of the episcleral venous plexus when balanced salt solution is injected into the anterior chamber) indicates that the trabecular bypass procedure is successful in accessing the downstream collector channels and aqueous veins. Fellman suggested that this wave can be induced with the aid of the irrigation and aspiration system: insert the irrigation and aspiration handpiece into the anterior chamber with the infusion turned off, then initiate maximal irrigation, creating a high-pressure gradient for balanced salt solution to surge from the anterior chamber through the Microstent into the canal and the venous collector system.

Another useful sign that Hydrus Microstent implantation is successful is the presence of blood reflux into the anterior chamber from the Microstent lumen under low-pressure conditions. When the IOP is lower than the pressure in the episcleral veins, retrograde blood flow through patent collector channels and Schlemm's canal can occur. Implantation of the Hydrus Microstent into Schlemm's canal further allows blood to flow from the Schlemm's canal into the anterior chamber. Grieshaber et al. showed that the postoperative IOP correlated with the quantity of blood reflux into Schlemm's canal in 28 eyes of African patients who underwent ab-externo canaloplasty [18]. In the same study, the patency of the collector channels was also checked by injecting fluorescein dye into Schlemm's canal using a flexible microcatheter. However, this technique required conjunctival peritomy, which would defeat the objective of the ab-interno approach and compromise the success of subsequent glaucoma filtration surgery if indicated. Ab-interno procedures for assessing the conventional outflow system spare the conjunctiva and can provide valuable information regarding Hydrus Microstent placement and the likelihood of success for Schlemm's canal surgery. For example, Saheb et al. injected dye through the inlet of the Microstent after implantation in order to assess the anatomy and patency of the distal outflow pathway [15]. Furthermore, high-resolution imaging of the conventional outflow system can also help to determine the optimal site of implantation. Kagemann et al. used spectral domain ocular coherence tomography (OCT) to noninvasively assess Schlemm's canal, collector channels, and the intrascleral venous plexus $[19,20]$. Further evolution of the imaging techniques and the introduction of OCT angiography may aid in planning optimal Hydrus Microstent placement and for predicting its probability of success.

\subsection{Patients}

Suitable patients for Hydrus Microstent implantation are those with primary openangle glaucoma and pseudoexfoliative glaucoma. Contraindications include angleclosure glaucoma and secondary glaucoma, such as neovascular, uveitic, traumatic, steroid-induced, and lens-induced glaucoma. Furthermore, patients who previously underwent argon laser trabeculoplasty, cyclo-destructive procedures, trabeculectomy, tube shunt implantation, or any incisional glaucoma surgical procedure are not ideal candidates for Hydrus Microstent implantation: possible changes in the Schlemm's canal after such interventions can make implantation challenging or 
impossible. Hence, the surgical outcomes of Hydrus Microstent implantation in such patients remain uncertain [1].

\subsection{Efficacy}

The performance of the Hydrus Microstent in reducing IOP was evaluated in the HYDRUS II study: a prospective, single-masked, randomized controlled clinical trial conducted in seven European centers [1]. Patients were randomized to either cataract surgery combined with Hydrus Microstent implantation or cataract surgery alone and then followed up for 2 years. In order to accurately determine the efficacy of the surgery, patients underwent a washout of their hypotensive medications, similar to the protocol in the Ocular Hypertension Treatment Study [21] (Table 5.1).

One year after Hydrus Microstent implantation in combination with cataract surgery, the mean washed-out IOP decreased significantly by $9.7 \mathrm{mmHg}$ (from $26.3 \mathrm{mmHg}$ to $16.6 \mathrm{mmHg}$ ) compared with preoperative IOP. This effect persisted for 2 years after surgery, with the mean washed out diurnal IOP only $0.3 \mathrm{mmHg}$ higher than that at 1 year at $16.9 \mathrm{mmHg}$. There was also a significant decrease in IOP at 1 year for eyes which underwent cataract surgery alone, with a mean reduction of $9.2 \mathrm{mmHg}$ (from $26.6 \mathrm{mmHg}$ to $17.4 \mathrm{mmHg}$ ). However, at 2 years, there was an increase in IOP to $19.2 \mathrm{mmHg}$ in eyes which underwent cataract surgery alone, and this was significantly higher than the 2-year IOP in eyes which underwent Hydrus Microstent implantation in combination with cataract surgery.

Combined Hydrus Microstent implantation and cataract surgery were also associated with a reduction in glaucoma medications, with $77.1 \%$ and $72.3 \%$ of patients requiring no medications to achieve target IOP at 1 year and 2 years after surgery, respectively. Amongst patients who underwent cataract surgery alone, the proportion of patients who were medication-free at 1 year and 2 years after surgery was significantly lower compared with those who underwent combined Hydrus Microstent implantation and cataract surgery, at $49 \%$ and $36.4 \%$, respectively. Moreover, patients who underwent combined Hydrus Microstent implantation and

Table 5.1 The efficacy of Hydrus ${ }^{\circledast}$ Microstent either as a standalone procedure or in combination with cataract surgery as reported in a number of studies [1, 8, 15, 22-24]

\begin{tabular}{l|l|l|l|l}
\hline Group & Procedure & Follow-up & Pre-OP IOP & Post-OP IOP \\
\hline Pfeiffer et al. [1] & Hydrus + Cataract Surgery & 2 years & $26.3 \mathrm{mmHg} *$ & $16.9 \mathrm{mmHg}^{*}$ \\
\hline Ahmed et al. [15] & Hydrus + Cataract Surgery & 6 months & $17.9 \mathrm{mmHg}$ & $15.3 \mathrm{mmHg}$ \\
\hline Fea et al. [22] & Hydrus + Cataract Surgery & 2 years & $19.4 \mathrm{mmHg}$ & $15.7 \mathrm{mmHg}$ \\
\hline $\begin{array}{l}\text { Al-Mugheiry et al. } \\
\text { [23] }\end{array}$ & Hydrus & 2 years & $18.1 \mathrm{mmHg}$ & $15.3 \mathrm{mmHg}$ \\
\hline Fea et al. [24] & Hydrus & 1 years & $23.1 \mathrm{mmHg}$ & $16.5 \mathrm{mmHg}$ \\
\hline Gandolfi et al. [8] & Hydrus & 2 years & $24.0 \mathrm{mmHg}$ & $15.0 \mathrm{mmHg}$ \\
\hline
\end{tabular}

*In the study by Pfeiffer et al., washout of glaucoma medication was performed in order to accurately determine the efficacy of Hydrus ${ }^{\circledR}$ Microstent implantation 
cataract surgery required only half the mean number of glaucoma medications at 2 years compared with those who underwent cataract surgery alone.

The results of the Hydrus II study are similar to that of other smaller case series. Ahmed et al. reported the 6-month outcomes of 28 eyes with mild-to-moderate primary open-angle glaucoma after combined phacoemulsification and Hydrus implantation [15]. Baseline IOP was $17.9 \pm 4.1 \mathrm{mmHg}$ with $2.4 \pm 1.0$ glaucoma medications, and washed-out IOP was $29.9 \pm 5.8 \mathrm{mmHg}$ before surgery. The IOP and the mean number of glaucoma medications at 6 months were significantly reduced to $15.3 \pm 2.3 \mathrm{mmHg}$ and $0.1 \pm 0.4$, respectively. Fea et al. reported the 2-year results of 92 eyes that underwent combined cataract surgery and Hydrus Microstent implantation [22]. This was a retrospective study with no washout of glaucoma medications. Nevertheless, a $20 \%$ reduction in the mean IOP from $19.4 \mathrm{mmHg}$ preoperatively to $15.7 \mathrm{mmHg}$ at 2 years after surgery was observed. In a single-center and single-surgeon observational study, Al-Mugheiry et al. reported the 2-year results of 25 eyes which underwent combined Hydrus Microstent implantation and cataract surgery. At the end of 2 years, the mean medicated IOP was reduced from $18.1( \pm 3.6) \mathrm{mmHg}$ to $15.3( \pm 2.2) \mathrm{mmHg}$ [23].

The HORIZON study was a 24-month prospective, multicenter, single-masked randomized controlled trial which compared the reduction in IOP and medication use in subjects who underwent combined cataract surgery and Hydrus Microstent implantation (HMS, $n=369$ ) with those who underwent cataract surgery alone (NMS, $n=187$ ). At 24 months, $77.3 \%$ of the HMS group eyes achieved $\geq 20 \%$ reduction in unmedicated modified diurnal IOP (MDIOP) compared with $57.8 \%$ of NMS group eyes (difference $=19.5 \%, 95 \%$ confidence interval $[\mathrm{CI}] 11.2 \%-27.8 \%$, $p<0.001)$. The mean \pm standard deviation decrease in unmedicated MDIOP was $-7.6 \pm 4.1 \mathrm{mmHg}$ in the HMS group and $-5.3 \pm 3.9 \mathrm{mmHg}$ in the NMS group at 24 months (difference $=2.3 \mathrm{mmHg}, 95 \% \mathrm{CI}-3.0$ to $-1.6, p<0.001$ ). The safety profile was similar in both groups with no serious ocular adverse events related to the microstent [24]. These results confirmed the findings of the HYDRUS II study, that the efficacy of combined phacoemulsification and Hydrus Microstent implantation in lowering the IOP and glaucoma medications was superior to phacoemulsification alone.

Although Hydrus Microstent implantation is typically performed in combination with cataract surgery, the device can also be implanted as a solo procedure. Fea et al. reported the 1-year outcomes of Hydrus Microstent implantation as a solo procedure in 31 eyes (20 phakic, 11 pseudophakic) with primary open-angle glaucoma [25]. One year after surgery, the mean IOP decreased by $6.6 \pm 5.6 \mathrm{mmHg}$ (from $23.1 \mathrm{mmHg}$ to $16.5 \mathrm{mmHg}$ ) and $47 \%$ of eyes were medication-free. This nonrandomized prospective study also compared the results of Microstent implantation with selective laser trabeculoplasty. At 1 year, IOP reduction was similar between Hydrus Microstent implantation and selective laser trabeculoplasty. However, eyes that underwent Hydrus Microstent implantation required significantly fewer medications to achieve target IOP compared with those which underwent selective laser trabeculoplasty, even though the eyes which underwent Hydrus Microstent implantation had more severe glaucoma. Gandolfi et al. reported the 
2-year results of stand-alone Hydrus Microstent implantation compared with canaloplasty [8]. There was a significant and similar IOP decrease in both groups, from $24 \pm 6 \mathrm{mmHg}$ to $15 \pm 3 \mathrm{mmHg}$ in eyes that underwent Hydrus Microstent implantation and from $26 \pm 4 \mathrm{mmHg}$ to $16 \pm 2 \mathrm{mmHg}$ in eyes which underwent canaloplasty. In the COMPARE study, Ahmed et al. randomized 152 eyes from 152 patients with open-angle glaucoma to standalone MIGS consisting of either one Hydrus Microstent or two iStent Trabecular Micro-Bypass devices in a prospective, multicenter, randomized clinical trial. At 12 months, the Hydrus group had a higher rate of complete success $(39.7 \%$ vs. $13.3 \%, p<0.001)$ and reduced medication use (difference $=-0.6$ medications, $p=0.004$ ), with more patients in the Hydrus group being medication-free (difference $=22.6 \%, p=0.0057$ ) [26].

\subsection{Safety}

Hydrus Microstent implantation is associated with a favorable safety profile [24, 27]. In the Hydrus II and HORIZON studies, the visual acuity of eyes that underwent combined cataract surgery with Hydrus Microstent implantation was similar to that of eyes after cataract surgery alone $[1,24]$. Adverse events, such as cornea punctate staining, erosion of the corneal epithelium, stromal edema, endothelial folds, anterior chamber cells, and flare, were generally mild. All these were observed in the early postoperative period, with resolution within 4 weeks. In the course of the follow-up in the Hydrus II study, the formation of peripheral anterior synechiae (Fig. 5.5) was observed in six eyes at 1 year and nine eyes at 2 years after Hydrus Microstent implantation. Serious ocular adverse events were rare and not attributed to the procedure in the opinion of the investigators (acute vitreomacular traction, anterior ischaemic optic neuropathy, retinal detachment, and macular edema) [1]. A study by Ahmed et al. reported a low incidence of corneal edema, hyphema, and peripheral anterior synechiae [15]. The presence of peripheral anterior synechiae was not related to an increase in IOP in the HORIZON study [24].

Fig. 5.5 Gonioscopy photograph showing peripheral anterior synechiae formation at the inlet of the Hydrus ${ }^{\circledR}$ Microstent. (Copyright Chelvin Sng, FRCSEd; reproduced with permission)

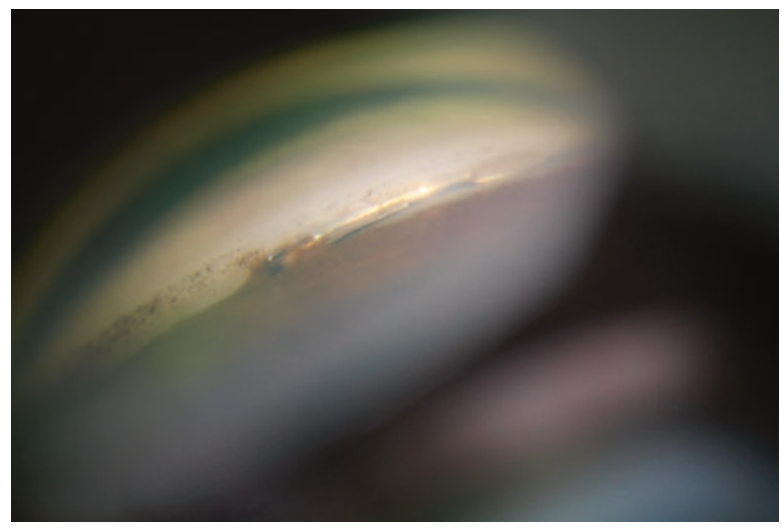


In a retrospective study by Fea et al., which included 92 eyes with combined Hydrus Microstent implantation and cataract surgery, one patient developed hyphema exceeding $2.0 \mathrm{~mm}$ in the early postoperative period and this resolved spontaneously without any sequelae [25]. The placement of the Hydrus Microstent was not satisfactory in two eyes and intraoperative repositioning was performed. Peripheral anterior synechiae without Microstent obstruction was observed in eight eyes, and iris occlusion of the device inlet occurred in one eye, which was treated with argon laser 8 months after the surgery. In one eye, the Microstent was malpositioned (outside Schlemm's canal) and the IOP was above target. This patient subsequently required trabeculectomy at 18 months. Al-Mugheiry et al. reported the outcomes of Hydrus Microstent implantation in 25 eyes by a surgeon with no prior surgical experience with the Microstent [23]. Despite the surgical inexperience, there were very few intraoperative complications. Hyphema occurred in two eyes during Microstent insertion. In one eye, the Microstent could not be fully inserted at the first attempt, but it was successfully inserted in another position at the second attempt. The postoperative adverse events on day 1 were mild-to-moderate anterior uveitis (12 eyes), mild corneal edema (7 eyes), microhyphema ( 7 eyes), or hyphema $>1.5 \mathrm{~mm}$ ( 2 eyes). In one eye with microhyphema, there was an associated IOP spike $(28 \mathrm{mmHg})$ and in one eye with a $2-\mathrm{mm}$ hyphema, there was a blood clot seen at the opening of the Microstent, but this resolved spontaneously after a week. There was no correlation between day 1 complications and outcome.

Stand-alone Hydrus Microstent implantation was also found to be safe for phakic eyes, with a low incidence of adverse events being reported. In the prospective study by Fea et al., IOP spikes occurred in 2 out of 31 eyes $(6.45 \%)$ on the first postoperative day [25]. After temporary treatment with systemic acetazolamide, the IOP was normalized in all eyes by the third postoperative day. There was a transient decrease in visual acuity in three eyes $(9.68 \%)$ on the first postoperative day, which was due to corneal edema secondary to an IOP spike in one eye and hyphema in two eyes. Visual acuity returned to baseline by 1 week after the surgery in all three cases. Gandolfi et al. reported that hyphema was the most common post-operative adverse event (4 out of 21 eyes) [8]. The hyphema cleared completely over a few days in all cases. An early postoperative IOP spike ( $\geq 30 \mathrm{mmHg}$ within the first $48 \mathrm{~h}$ ) occurred in one eye after Hydrus Microstent implantation. Peripheral anterior synechiae developed in four eyes during follow-up, which was treated with a YAG laser procedure. Ahmed et al. reported that there was no significant difference in adverse events at one year between study eyes which underwent standalone Hydrus Microstent implantation compared with those which underwent implantation of two iStent Trabecular Micro-Bypass devices in the COMPARE study. Two subjects in the two iStent group required subsequent glaucoma surgery due to uncontrolled IOP despite maximum medical therapy, while none of the subjects in the Hydrus group required additional incisional glaucoma surgery. Ocular adverse events in both groups were mostly mild and transient [26]. 
In summary, Hydrus Microstent implantation may be associated with transient IOP spikes, hyphema, stent malposition or obstruction and the development of peripheral anterior synechiae. IOP spikes occur mostly in the early postoperative period and can be easily managed with glaucoma medications. Bleeding in the anterior chamber can vary from a circulating or micro-hyphema to a large hyphema. This is typically transient and self-resolving within a few days or weeks. Microstent malposition is associated with impaired stent function and surgical intervention may be required in these cases. Development of peripheral anterior synechiae is relatively common postoperatively, but is without clinical significance in most of the cases. However, synechial formation at the Hydrus Microstent inlet can result in stent obstruction, and this may be relieved by laser treatment in some cases. Clinically significant and long-standing hypotony or other potentially sight-threatening complications have not been described so far with Hydrus Microstent implantation, as its capacity to reduce IOP is limited by episcleral venous pressure.

\subsection{Conclusion}

Hydrus Microstent implantation in combination with cataract surgery or as a solo procedure is safe and effective for the treatment of primary open-angle glaucoma. This Schlemm's canal scaffold lowers IOP to the mid-teens and reduces glaucoma medication use for up to 2 years. Hence, it is promising as a long-term treatment modality for patients with mild to moderate primary open-angle glaucoma.

\section{References}

1. Pfeiffer N, et al. A randomized trial of a Schlemm's canal microstent with phacoemulsification for reducing intraocular pressure in open-angle glaucoma. Ophthalmology. 2015;122(7):1283-93.

2. Johnstone MA, et al. Effects of a Schlemm canal scaffold on collector channel ostia in human anterior segments. Exp Eye Res. 2014;119:70-6.

3. Gulati V, et al. A novel 8-mm Schlemm's canal scaffold reduces outflow resistance in a human anterior segment perfusion model. Invest Ophthalmol Vis Sci. 2013;54(3):1698-704.

4. Beeley NR, et al. Development, implantation, in vivo elution, and retrieval of a biocompatible, sustained release subretinal drug delivery system. J Biomed Mater Res A. 2006;76(4):690-8.

5. Ko GY, et al. Obstruction of the lacrimal system: treatment with a covered, retrievable, expandable nitinol stent versus a lacrimal polyurethane stent. Radiology. 2003;227(1): $270-6$.

6. Olson JL, Velez-Montoya R, Erlanger M. Ocular biocompatibility of nitinol intraocular clips. Invest Ophthalmol Vis Sci. 2012;53(1):354-60.

7. Grierson I, et al. A novel Schlemm's canal scaffold: histologic observations. J Glaucoma. 2015;24(6):460-8. 
8. Gandolfi SA, et al. Comparison of surgical outcomes between canaloplasty and Schlemm's canal scaffold at 24 Months' follow-up. J Ophthalmol. 2016;2016:3410469.

9. Johnstone MA, Grant WG. Pressure-dependent changes in structures of the aqueous outflow system of human and monkey eyes. Am J Ophthalmol. 1973;75(3):365-83.

10. Johnstone MA, Grant WM. Microsurgery of Schlemm's canal and the human aqueous outflow system. Am J Ophthalmol. 1973;76(6):906-17.

11. Yuan F, et al. Mathematical modeling of outflow facility increase with trabecular meshwork bypass and Schlemm Canal dilation. J Glaucoma. 2016;25(4):355-64.

12. Camras LJ, et al. A novel Schlemm's canal scaffold increases outflow facility in a human anterior segment perfusion model. Invest Ophthalmol Vis Sci. 2012;53(10):6115-21.

13. Hays CL, et al. Improvement in outflow facility by two novel microinvasive glaucoma surgery implants. Invest Ophthalmol Vis Sci. 2014;55(3):1893-900.

14. Moses RA, et al. Schlemm's canal: the effect of intraocular pressure. Invest Ophthalmol Vis Sci. 1981;20(1):61-8.

15. Saheb H, Ahmed II. Micro-invasive glaucoma surgery: current perspectives and future directions. Curr Opin Ophthalmol. 2012;23(2):96-104.

16. Bill A. Some aspects of aqueous humour drainage. Eye (Lond). 1993;7(Pt 1):14-9.

17. Fellman RL, Grover DS. Episcleral venous fluid wave: intraoperative evidence for patency of the conventional outflow system. J Glaucoma. 2014;23(6):347-50.

18. Grieshaber MC, et al. Clinical evaluation of the aqueous outflow system in primary open-angle glaucoma for canaloplasty. Invest Ophthalmol Vis Sci. 2010;51(3):1498-504.

19. Kagemann L, et al. Identification and assessment of Schlemm's canal by spectral-domain optical coherence tomography. Invest Ophthalmol Vis Sci. 2010;51(8):4054-9.

20. Kagemann L, et al. 3D visualization of aqueous humor outflow structures in-situ in humans. Exp Eye Res. 2011;93(3):308-15.

21. Mansberger SL, et al. Reduction in intraocular pressure after cataract extraction: the ocular hypertension treatment study. Ophthalmology. 2012;119(9):1826-31.

22. Fea AM, Rekas M, Au L. Evaluation of a Schlemm canal scaffold Microstent combined with phacoemulsification in routine clinical practice: two-year multicenter study. J Cataract Refract Surg. 2017;43(7):886-91.

23. Al-Mugheiry TS, et al. Microinvasive glaucoma stent (MIGS) surgery with concomitant phakoemulsification cataract extraction: outcomes and the learning curve. J Glaucoma. 2017;26(7):646-51.

24. Samuelson TW, Chang DF, Marquis R, et al. A Schlemm canal microstent for intraocular pressure reduction in primary open-angle glaucoma and cataract: the HORIZON study. Ophthalmology. 2019;126:29-37.

25. Fea AM, et al. Hydrus Microstent compared to selective laser trabeculoplasty in primary open angle glaucoma: one year results. Clin Exp Ophthalmol. 2017;45(2):120-7.

26. Ahmed IIK, Fea A, Au L, et al. A prospective randomized trial comparing Hydrus and iStent microinvasive glaucoma surgery implants for standalone treatment of open-angle glaucoma: the COMPARE study. Ophthalmology. 2020;127:52-61.

27. Yook E, Vinod K, Panarelli JF. Complications of micro-invasive glaucoma surgery. Curr Opin Ophthalmol. 2018;29:147-54. 
Open Access This chapter is licensed under the terms of the Creative Commons Attribution 4.0 International License (http://creativecommons.org/licenses/by/4.0/), which permits use, sharing, adaptation, distribution and reproduction in any medium or format, as long as you give appropriate credit to the original author(s) and the source, provide a link to the Creative Commons license and indicate if changes were made.

The images or other third party material in this chapter are included in the chapter's Creative Commons license, unless indicated otherwise in a credit line to the material. If material is not included in the chapter's Creative Commons license and your intended use is not permitted by statutory regulation or exceeds the permitted use, you will need to obtain permission directly from the copyright holder. 